\title{
Simple, adjustable beam splitting element for differential interferometers based on photoelastic birefringence of a prismatic bar
}

\author{
S. R. Sanderson ${ }^{\text {a) }}$ \\ Graduate Aeronautical Laboratories, California Institute of Technology, Pasadena, California 91125
}

(Received 6 June 2005; accepted 9 October 2005; published online 23 November 2005)

\begin{abstract}
We examine the prototypical Toepler optical arrangement for the visualization of phase objects and consider the effect of different contrast elements placed at the focus of the source. In particular, Wollaston prism beam splitting elements based on the crystallographic birefringence of calcite or quartz find application in differential interferometry systems based on the Toepler arrangement. The focus of the current article is a simple low cost alternative to the Wollaston prism that is realized by inserting a prismatic bar constructed of a photoelastic material into the optical path. It is shown that, under the action of an applied bending moment, the prismatic bar functions as a first-order approximation to a Wollaston prism. Results are derived for the divergence angle of the beam splitter for orthogonally polarized rays. The implementation of a practical device is discussed and representative experimental results are presented, taken from the field of shock wave visualization in supersonic flow. (C) 2005 American Institute of Physics. [DOI: 10.1063/1.2132271]
\end{abstract}

\section{INTRODUCTION}

Figure 1 illustrates the conventional Toepler arrangement that represents the prototypical symmetric optical layout for the visualization of phase objects. Systems based on this arrangement find wide application in the fields of compressible flow visualization, convective heat transfer, and optical inspection systems (see Refs. 1 and 2). For simplicity, the focal lengths of the source and objective lenses are chosen to be equal and a light source is placed at the focus of the beam forming lens that produces a collimated beam passing through the test section. The test section lies midway between the beam forming and objective lenses and here the objective lens is placed two focal lengths from the test section so that the test section is imaged at unity magnification onto the detector plane, also two focal lengths distant from the objective lens.

Two sets of rays are shown in Fig. 1 according to elementary principles of paraxial optics; the extreme rays emitted by the light source and subsequently brought to focus midway between the objective lens and the detector plane, and the extreme rays potentially collected by the objective lens that have passed though the midpoint of the test section (these rays subtend a vanishing small angle as the source size tends to zero and for clarity only the set of rays passing through the center point of the test section are shown).

Phase objects placed in the test section cause an optical phase shift in the rays passing through the test section, and refractive index gradients within the phase object cause an additional angular deflection of the rays passing through the test section. In the absence of a contrast producing element at the image of the source and with the detector placed at an

a) Present address: General Electric Industrial-Security, 12345 SW Leveton Drive, Tualatin, OR 97062 image plane of the test section (i.e., focused on the midplane of the test section), the deflected and phase-shifted rays are brought back to focus from their deflected state at the detector plane yielding a uniformly illuminated detector. Shadowgraph images may be produced by de-focusing the system so that the detector no longer lies at the image plane of the test section. Schlieren systems retain the focused configuration, and phase objects are visualized by introducing a contrast element (typically a knife edge) that modulates the intensity of the rays when they are deflected at the image of the source.

\section{DIFFERENTIAL INTERFEROMETRY}

Interferometric methods are of great value for quantitative flow visualization, however, the cost of precision optical elements poses a significant barrier. A differential interferometer relies on the use of a beam splitting element placed at the image of the source. When inserted at the contrast element location in the Toepler optical layout the function of the beam splitter, rather than to split incident rays, is to recombine slightly offset rays that have passed through the test section. The resulting constructive and destructive interferences between these rays then produce interference fringes at the detector (here we assume the use of a single prism with a coherent light source).

Generally, the fringe order produced in differential interferometry is proportional to the difference in the refractive index integrated along the two beam paths though the test section. Specifically for compressible flow visualization experiments, the fringe order, $i$, of the interference produced between the two rays is ${ }^{1,2}$ 


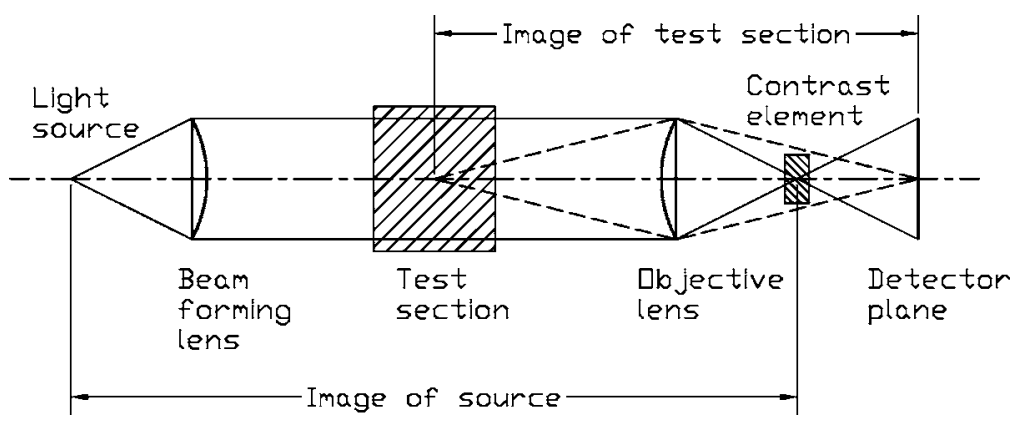

FIG. 1. Prototypical optical layout for Toepler visualization system. The arrangement is symmetrical with the source and objective lenses placed two focal lengths from the test section. The test section is imaged full size onto the detector, also two focal lengths from the objective lens. The solid rays show the envelope of the source beam. The dashed rays indicate the undistorted imaging of all rays passing through a given point in the test section onto the detector (these extreme rays do not exist for a small source).

$$
i=\frac{\Delta \rho L K}{\lambda},
$$

where $\Delta \rho$ is the density difference between the two ray paths, $L$ is the path length through the test section, $K$ is the Gladstone-Dale constant for the gas, and $\lambda$ is the wavelength of the light source.

\section{WOLLASTON PRISM}

A Wollaston prism is commonly used to perform the beam splitting function in a differential interferometer. To understand the behavior of the new beam splitting element that is proposed here, it is helpful to briefly review the familiar behavior of the existing device. The Wollaston prism owes its behavior to the birefringent optical properties of the calcite or quartz materials from which it is commonly constructed. A prism is formed with a split plane at angle $\alpha$ with respect to the entrance and exit faces (see Fig. 2). Along the split plane the optical axes of two prism halves are rotated by $90^{\circ}$ so that the ordinary, $o$, and extraordinary, $e$, rays are reversed in the two halves of the prism. An incident linearly polarized ordinary ray therefore becomes an extraordinary ray in the second half of the prism, and vice versa for an incident extraordinary ray. We can understand the effect of this arrangement by applying Snell's law at each of the interfaces for each of the rays as shown in Fig. 2. Equations (2) and (5) apply at the entrance surface of the prism, Eqs. (3) and (6) apply at the split plane where the refractive indices reverse for the two rays, respectively, and Eqs. (4) and (7) apply where the diverging rays exit the prism,

$$
\begin{aligned}
& n_{\infty} \theta_{i}=n_{o} \theta_{1 o}, \\
& n_{o}\left(\alpha-\theta_{1 o}\right)=n_{e}\left(\alpha-\theta_{2 e}\right), \\
& n_{e} \theta_{2 e}=n_{\infty} \theta_{3-},
\end{aligned}
$$

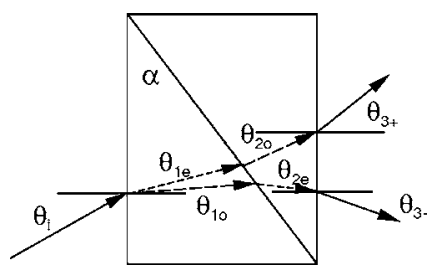

FIG. 2. Prototypical optical layout for a Wollaston prism with split plane angle $\alpha$. $-\ldots$ indicates the ordinary rays and --- indicates the extraordinary rays that exchange orientations at the split plane due to the $90^{\circ}$ rotation of the optical axes in the two half prisms.

$$
\begin{aligned}
& n_{\infty} \theta_{i}=n_{e} \theta_{1 e}, \\
& n_{e}\left(\alpha-\theta_{1 e}\right)=n_{o}\left(\alpha-\theta_{2 o}\right), \\
& n_{o} \theta_{2 o}=n_{\infty} \theta_{3+},
\end{aligned}
$$

where the $n$ are the refractive indices, subscripts $o$ and $e$ denote ordinary and extraordinary rays, respectively, and the subscript $\infty$ refers to the surrounding optical medium. The various ray angles, $\theta$, are specified in Fig. 2. After manipulation we have the emergence angles for the two rays,

$$
\begin{aligned}
& \theta_{3+}=\alpha \frac{n_{o}-n_{e}}{n_{\infty}}+\theta_{i}, \\
& \theta_{3-}=-\alpha \frac{n_{o}-n_{e}}{n_{\infty}}+\theta_{i},
\end{aligned}
$$

and the resulting beam divergence angle,

$$
\Delta \theta=\theta_{3+}-\theta_{3-}=2 \alpha \frac{n_{o}-n_{e}}{n_{\infty}},
$$

that depends on the split plane angle of the prism and the birefringence of the prism material relative to the surrounding optical medium. We see that the function of a Wollaston prism is therefore to take a linearly polarized beam and to split it into two orthogonally polarized rays that diverge by a small angle, $\Delta \theta$.

Any linearly polarized ray incident on the prism may be decomposed into two orthogonal, linearly polarized components, aligned with the ordinary and extraordinary rays of the birefringent material. The beam splitting element is typically located between crossed polarizing elements (e.g., Ref. 1) oriented at $45^{\circ}$ to the optical axes of the prism so that the ordinary and extraordinary rays will have equal magnitudes. A second polarizing element behind the beam splitter, also at $45^{\circ}$ to the principal optical axes of the beam splitter, serves to select the common components of the ordinary and extraordinary rays and recombine them into a single linearly polarized ray exiting the system.

\section{RESULTS FROM PHOTOELASTICITY THEORY}

Before proceeding we introduce some key results from the theory of photoelasticity (see, e.g., Refs. 3-5). Isotropic optical materials can be characterized by a single value of refractive index. Anisotropic optical media, however, require definition of a refractive that varies with the direction of propagation of a ray through the material. This variation in the refractive index may be represented by Fresnel's ellip- 


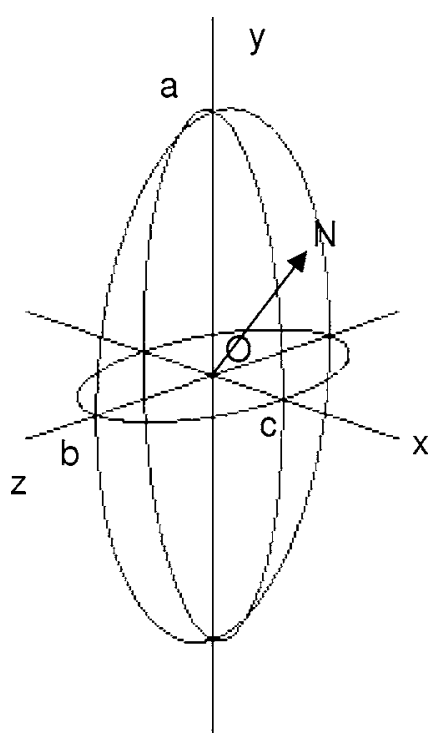

FIG. 3. Fresnel's ellipsoid for anisotropic optical media with principal optical axes $a, b, c$ aligned with axes $x, y, z$. Vector $\mathbf{O N}$ indicates the direction of propagation of a hypothetical ray.

soid (see Fig. 3). If we consider the propagation of a ray $\mathbf{O N}$ relative to the material as shown in Fig. 3 and construct a plane normal to ray $\mathbf{O N}$, also containing the origin, then the intersection of this plane and the ellipsoid defines an ellipse. Each point on the ellipse represents the refractive index for rays of varying angle of linear polarization, propagating in direction $\mathbf{O N}$. The major and minor axes of the ellipse correspond to the slow and fast axes, respectively, for rays propagating in direction $\mathbf{O N}$.

Maxwell went on to show that the principal optical axes correspond to the axes of the principal stresses for a photoelastic material in a generally three-dimensional state of stress and that the principal values of the refractive indices $n_{a, b, c}$ are related to the principal values of the stress $\sigma_{1,2,3}$ according to the stress-optic law

$$
\begin{aligned}
& n_{a}-n_{i}=C_{1} \sigma_{1}-C_{2}\left(\sigma_{2}+\sigma_{3}\right), \\
& n_{b}-n_{i}=C_{1} \sigma_{2}-C_{2}\left(\sigma_{1}+\sigma_{3}\right), \\
& n_{c}-n_{i}=C_{1} \sigma_{3}-C_{2}\left(\sigma_{1}+\sigma_{2}\right) .
\end{aligned}
$$

The photoelastic constants, $C_{1}$ and $C_{2}$, are physical properties for a particular material and $n_{i}$ is the isotropic refractive index of the material in a stress-free state.

\section{PHOTOELASTIC BEAM SPLITTING ELEMENT}

We now explore one particular stress distribution that produces a gradient of birefringence that has utility in optical apparatus. Specifically we consider the stress state in a prismatic bar subjected to an applied bending moment (see Fig. 4). Clearly, different stress states may be conceived that produce equivalently useful results. The exact solution for the stress state in the bar of Fig. 4 is known from elasticity theory to be ${ }^{6}$

$$
\sigma_{1}=\sigma_{x}=\frac{M_{z} y}{I_{z z}},
$$

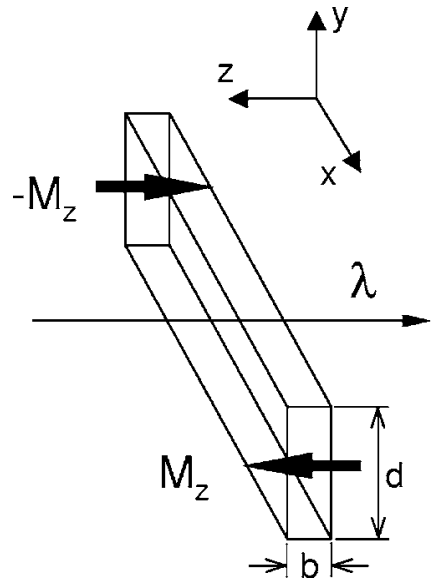

FIG. 4. Configuration of prismatic bar with breadth $b$ and depth $d$ under action of applied bending moment $M_{z}$. The optical path through the center of the bar for a ray with wavelength $\lambda$ is indicated. The stress state in the optical center section of the bar is independent of the length of the bar.

$$
\sigma_{2}=\sigma_{3}=0,
$$

where $M_{z}$ is the applied bending moment, $y$ is the distance from the centerline, and $I_{z z}$ is the second moment of area of the bar cross section about the $z$ axis.

If this stress distribution is substituted into the stressoptic law [Eqs. (11)-(13)] then we obtain the spatial distribution of the principal indices of refraction for the bar configuration

$$
\begin{aligned}
& n_{a}-n_{i}=C_{1} \frac{M_{z} y}{I_{z z}}, \\
& n_{b}-n_{i}=-C_{2} \frac{M_{z} y}{I_{z z}}, \\
& n_{c}-n_{i}=-C_{2} \frac{M_{z} y}{I_{z z}} .
\end{aligned}
$$

Referring back to the discussion of Fresnel's ellipsoid, we may now construct the ellipse that represents the variation of refractive index with polarization angle for a ray propagating parallel to the $z$ axis (see Fig. 5).

Following the pattern of analysis established for the Wollaston prism, we consider the paraxial behavior of a ray passing through the stressed prismatic bar (see Fig. 6). In the same way that the curvature and resultant angular deflection of a ray traversing the test section of a Schlieren system depend on the gradient of refractive index along the optical path, so too the angular deflection of the ray propagating

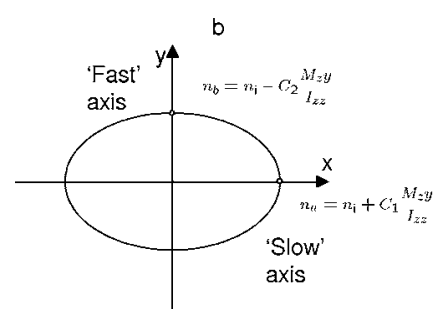

FIG. 5. Section through Fresnel ellipsoid in direction of beam propagation for photoelastic beam splitter. 


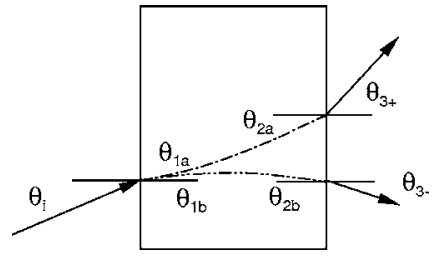

FIG. 6. Paraxial ray propagation through proposed photoelastic beam splitter under the action of a linear axial stress gradient. The slow and fast rays refract in opposite directions due the opposite signs of the stress-induced refractive index gradients.

through the prismatic bar depends on the stress-induced refractive index gradient within the bar. Specifically the angular deflection is given by ${ }^{1}$

$$
\epsilon \approx \frac{b}{n} \frac{\partial n}{\partial y},
$$

where $b$ is the thickness of the bar, and $y$ is the distance of the ray path from the centerline of the bar. Due to the stressinduced birefringence, orthogonally polarized rays experience refractive index gradients of opposite signs,

$$
\frac{\partial n_{a, b}}{\partial y}= \pm C_{1,2} \frac{M_{z}}{I_{z z}}
$$

and curve and deflect in opposite directions while traversing the bar,

$$
\epsilon_{a, b} \approx \frac{b}{n_{a, b}} \frac{\partial n_{a, b}}{\partial y}=\frac{b}{n_{i} \pm C_{1,2} M_{z} y / I_{z z}}\left( \pm C_{1,2}\right) \frac{M_{z}}{I_{z z}}
$$

In the same way that we applied Snell's law at each of the three interfaces of the Wollaston prism, for each of the orthogonally polarized components, we replace Eqs. (2)-(7) with Eqs. (22)-(26),

$$
\begin{aligned}
& n_{\infty} \theta_{i}=n_{1 a} \theta_{1 a}, \\
& n_{2 a} \theta_{2 a}=n_{\infty} \theta_{3+}, \\
& n_{\infty} \theta_{i}=n_{1 b} \theta_{1 b}, \\
& n_{2 b} \theta_{2 b}=n_{\infty} \theta_{3-}, \\
& \theta_{2 a, b}=\theta_{1 a, b}+\frac{b}{n_{i} \pm C_{1,2} M_{z} y / I_{z z}}\left( \pm C_{1,2}\right) \frac{M_{z}}{I_{z z}},
\end{aligned}
$$

where the results for the refraction at the prism split plane have been replaced by Eq. (26) that describe the refraction of the slow and fast rays by the refractive index gradient within the photoelastic prismatic bar. Note also the notation $n_{1,2 a, b}$ indicating the specific values of $n_{a, b}(y)$ for the $a$ and $b$ rays at the locations where they cross the entrance and exit faces of the photoelastic bar. Again, after manipulation and linearizing according to $\theta_{i} \ll 1$ and $C_{1,2}\left(M_{z} b / I_{z z}\right) \ll 1$ we obtain the paraxial approximations for the departure angles of the two orthogonally polarized rays from the prismatic bar,

$$
\theta_{3 \pm} \approx \theta_{i} \pm \frac{C_{1,2}}{n_{\infty}} \frac{M_{z} b}{I_{z z}}
$$

and the resulting beam divergence angle,

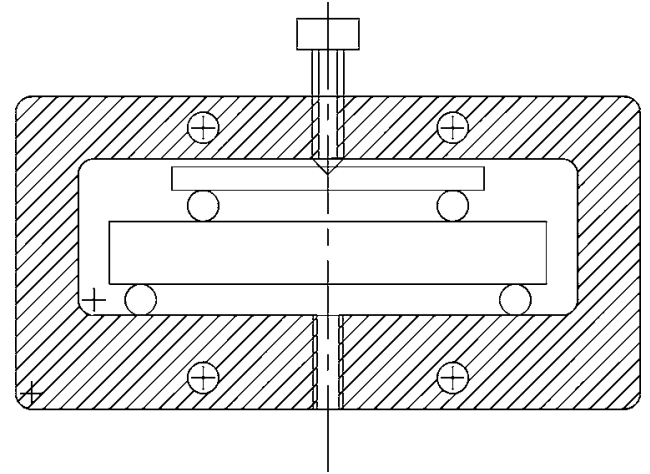

FIG. 7. Loading fixture for prismatic bar.

$$
\Delta \theta=\theta_{3+}-\theta_{3-} \approx \frac{C_{1}+C_{2}}{n_{\infty}} \frac{M_{z} b}{I_{z z}} .
$$

The beams therefore emerge from the prismatic bar at an angle that is deflected from the angle of incidence, and the divergence angle depends on the photoelastic constants for the material, the applied bending stress and the surrounding optical medium. Comparing this with the behavior of the Wollaston prism given by Eq. (10), where the divergence depends on the birefringent characteristics of the prism material and split plane angle, we see that in the limit of paraxial behavior and for low applied stress levels, the photoelastic prismatic bar with applied bending moment functions as a first-order approximation to the behavior of a Wollaston prism.

\section{IMPLEMENTATION OF PROTOTYPE BEAM SPLITTER}

Many materials exhibit stress-induced birefringence, with some polymer materials possessing especially high photoelastic coefficients. Polycarbonate (produced commercially under various trade names: Hyzod, Lexan, Makrolon, and Tuffak) possesses particularly high photoelastic coefficients, and is available in optically finished sheets of various thicknesses. The sheets are provided with self-adhesive protective backings that facilitate fabrication of the polycarbonate material into the desired configuration. For the current experiments a $6.35 \mathrm{~mm}$ thickness was found to provide adequate sensitivity.

The loading fixture shown in Figs. 7 and 8 was used to induce a stress state of pure bending in the photoelastic material. The loading fixture consists of a surrounding aluminum frame, the photoelastic material, a loading bar, an adjustable loading screw, and four short dowel pins that provide point contacts between the photoelastic material, frame, and loading bar. The force applied by the loading screw is transferred into the photoelastic material via the upper set of point contacts, and is resisted by the lower set of point contacts. Curved notches are cut into the upper and lower surfaces of the photoelastic material and into the lower surface of the loading bar to precisely locate the dowels. A countersunk hole is cut into the top surface of the loading bar to accept the chamfered end of the loading bolt. Since the two upper dowels are symmetrically arranged with respect to the loading bolt, the load applied to each end of the loading 


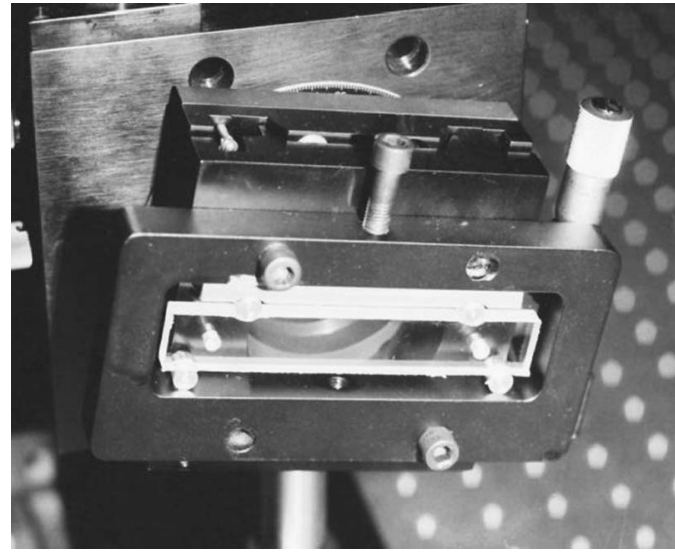

FIG. 8. Prototype differential interferometer showing the photoelastic beam splitter mounted on a translation stage for manipulation of the background fringe order. Ancillary optical elements (crossed polarizers, laser line interference filter, and spatial filter) have been removed for clarity. The slight rotation of the photoelastic bar is to align the sensitive axis of the interferometer with image rotation that occured as a result of complex out-of-plane folding of the optical path in this particular mirror-based implementation of the Toepler system. The curved registration notches used to locate the dowel pins are visible in the photoelastic bar.

bar is exactly half of the load applied by the loading bolt. Since the lower dowels are also symmetrical about the loading bolt, and since the horizontal spacing between the dowels on each side of the photoelastic material are identical, the photoelastic material is loaded in a state of pure bending, consistent with the assumed stress distribution that underlies Eq. (28) (localized stresses at the loading points vanish in the optical midsection of the bar according to St. Venant's principle).

Wollaston prisms are expensive due to the large optical quality crystals required for their fabrication. The divergence angle of a Wollaston prism and the resulting sensitivity of a differential interferometer are fixed for a prism cut at a specified angle $\alpha$. Toepler systems, whereby the split plane of the prism lies at the focus of a high-energy pulsed laser source, predispose the prism to optical damage. The photoelastic beam splitter has been shown to approximate the gradient of net crystallographic birefringence produced by a Wollaston prism with a functionally equivalent distribution of photoelastic birefringence, induced by a linear stress gradient across the prismatic bar. Optics of only nominal quality are sufficient for differential interferometry provided the offset between the differential beams remains small. The overall material and fabrication costs for a differential interferometer are therefore considerably reduced by the current device that mitigates the remaining expense contributed by the Wollaston prism. Adjustable sensitivity obviates the need for multiple Wollaston prisms to cover a useful range of divergence angles for the interferometer. The low cost of replacement and the absence of a material discontinuity at the laser focus provide advantages with respect to optical damage.

\section{EXPERIMENTAL RESULTS}

We have used this optical configuration to visualize a category of shock wave interaction flow fields ${ }^{7}$ that were

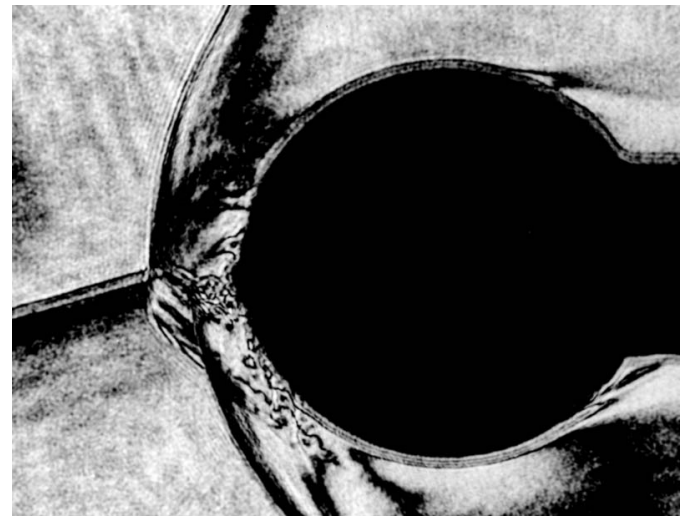

FIG. 9. Example differential interferogram obtained using prototype photoelastic beam splitter.

originally categorized by Edney ${ }^{8}$ (see also Ref. 9 for discussion of the application of holographic interferometry to the same flow field). Figure 9 is an example of the results obtained. We observe an incident shock wave entering the image from the left, and interacting with the bow shock wave ahead of a blunt body in the supersonic flow. The resulting interaction jet structure, embedded secondary shock waves, and convected vortical structures above and below the jet are clearly visualized along with the separation points downstream of the body. We also observe the vertically displaced double images of features in the image that are characteristic of differential interferometers. The type of image shown here is an infinite fringe differential interferogram and the usual manipulations of polarizer angles to influence fringe contrast, lateral displacement of the beam splitter to determine the background fringe order, and axial displacement of the beam splitter to operate in a finite fringe configuration are possible (see Ref. 1 for discussion).

Note that we have described a scenario where the photoelastic bar is placed at the image of the source, and so functions as a beam splitter in a differential interferometer. Equivalently, by placing the photoelastic bar at the test section location (along with the crossed polarizers), the bar element itself is imaged onto the detector. This exactly mimics the polariscope configuration used in photoelasticity studies and so the gradient of birefringence across the bar becomes visible and measurable for calibration purposes in the form of horizontal fringes.

${ }^{1}$ W. Merzkirch, Flow Visualization, 2nd ed. (Academic, New York, 1987). ${ }^{2}$ G. S. Settles, Schlieren and Shadowgraph Techniques (Springer, New York, 2001).

${ }^{3}$ J. W. Dally and W. F. Riley, Experimental Stress Analysis (McGraw-Hill, New York, 1991).

${ }^{4}$ P. S. Theocaris and E. E. Gdoutos, Matrix Theory of Photoelasticity (Springer, New York, 1979).

${ }^{5}$ E. G. Coker and L. N. G. Filon, A Treatise on Photoelasticity (Cambridge University Press, Cambridge, 1931).

${ }^{6}$ S. P. Timoshenko and J. N. Goodier, Theory of Elasticity (McGraw-Hill, New York, 1970).

${ }^{7}$ S. R. Sanderson and B. Sturtevant, Proceedings of the 19th International Symposium on Shock Waves, Marseille, 1993, edited by R. Brun and L. Z. Dumitrescu (Springer, Berlin, 1995), pp. 69-74.

${ }^{8}$ B. E. Edney, AIAA J. 6, 15 (1968).

${ }^{9}$ S. R. Sanderson, H. G. Hornung, and B. Sturtevant, J. Fluid Mech. 516, 1 (2004) 